\title{
O desenvolvimento de tumores cerebrais em idade pediátrica
}

\author{
Development of brain tumors in pediatric age
}

Desarrollo de tumores cerebrales en la edad pediátrica

Elisângela Faustino Farias da Costa ${ }^{1 *}$, Silvia Cristina Fürbringer e Silva ${ }^{2}$.

\section{RESUMO}

Objetivo: Reunir informações recentes sobre tumores cerebrais na idade pediátrica, trazendo dados sobre os tipos mais comuns, descobertas sobre seu desenvolvimento, consequências em qualidade de vida, tratamentos e diagnósticos. Revisão bibliográfica: Os tumores malignos pediátricos geralmente afetam as células do sistema hematopoiético e os tecidos de sustentação, o que se explica por serem predominantemente de natureza embrionária, oriundos de células indiferenciadas, diferentemente do que ocorre com o câncer em adultos, que costuma afetar as células do epitélio. Os tumores cerebrais malignos ocupam entre o primeiro e o terceiro lugar em incidência e mortalidade por câncer em crianças, dependendo do país, e são a principal causa de morte por câncer nessa população. Entre os tumores cerebrais mais comuns na população pediátrica estão o meduloblastoma e o glioma. O uso de FET-PET parece ser mais preciso na diferenciação e avaliação de tumores cerebrais na idade pediátrica do que a ressonância magnética, devendo ser indicada quando houver dúvidas na identificação das lesões. Considerações finais: Ainda pouco se sabe sobre a etiologia do tumor cerebral na infância. Também se faz necessário um melhor direcionamento para os tratamentos desses tipos de câncer, o que vem sendo desenvolvido através de terapêuticas epigenéticas.

Palavras-chave: Tumor cerebral, Crianças, Pediatria.

\begin{abstract}
Objective: To gather recent information on brain tumors in pediatric age, bringing data on the most common types, discoveries about their development, consequences on quality of life, treatments, and diagnoses. Bibliographic review: Pediatric malignant tumors generally affect the cells of the hematopoietic system and the supporting tissues, which is explained by the fact that they are predominantly embryonic in nature, originating from undifferentiated cells, unlike what occurs with cancer in adults, which usually affects the epithelial cells. Malignant brain tumors occupy between first and third place in cancer incidence and mortality in children, depending on the country, and are the main cause of cancer death in this population. Among the most common brain tumors in the pediatric population are medulloblastoma and glioma. The use of FET-PET seems to be more accurate in differentiating and evaluating brain tumors in pediatric age than MRI and should be indicated when there are doubts in the identification of lesions. Final considerations: Little is known about the etiology of childhood brain tumor. Better targeting for the treatment of these types of cancer is also needed, which has been developed through epigenetic therapies.
\end{abstract}

Key words: Brain tumor, Children, Pediatrics.

\section{RESUMEN}

Objetivo: Recoger información reciente sobre los tumores cerebrales en la edad pediátrica, aportando datos sobre los tipos más comunes, descubrimientos sobre su desarrollo, consecuencias en la calidad de vida, tratamientos y diagnósticos. Revisión bibliográfica: Los tumores malignos pediátricos suelen afectar las células del sistema hematopoyético y los tejidos de sostén, lo que se explica por el hecho de que son de naturaleza predominantemente embrionaria, originados a partir de células indiferenciadas, a diferencia de lo que ocurre con el cáncer en adultos, que suele afectar a las células epiteliales. Los tumores cerebrales malignos ocupan entre el primer y tercer lugar en la incidencia y mortalidad por cáncer en niños, según los países, y son la principal causa de muerte por cáncer en esta población. Entre los tumores cerebrales más

\footnotetext{
${ }^{1}$ Universidade Brasileira (IBRATI), São Caetano do Sul - SP. *E-mail: elisangela-farias28@hotmail.com

2 Universidade de São Paulo (SOPATI), Bela Vista - SP.
} 
comunes en la población pediátrica se encuentran el meduloblastoma y el glioma. El uso de FET-PET parece ser más preciso para diferenciar y evaluar tumores cerebrales en edad pediátrica que la RM, y debe estar indicado cuando existen dudas en la identificación de lesiones. Consideraciones finales: Se sabe poco sobre la etiología del tumor cerebral infantil. También existe la necesidad de una mejor orientación para el tratamiento de estos tipos de cáncer, que se ha desarrollado mediante terapias epigenéticas.

Palabras clave: Tumor cerebral, Niños, Pediatría.

\section{INTRODUÇÃO}

A incidência de subtipos de câncer varia entre crianças e adultos. Por exemplo, os tumores cerebrais malignos estão entre as principais causas de morte por câncer em pacientes mais jovens, enquanto em populações mais velhas são o câncer de pulmão e brônquios (JEAN-QUARTIER C, et al., 2021). Na população neonatal, entretanto, os tumores do sistema nervoso central (SNC) são relativamente raros. Frequentemente, eles carregam um prognóstico sombrio, em parte devido às opções terapêuticas limitadas disponíveis para recém-nascidos e à biologia única desses tumores em comparação com aqueles observados em bebês mais velhos e crianças (SHAHAB S e FANGUSARO J, 2021).

Os tumores cerebrais pediátricos são o tumor sólido mais comum na população pediátrica (YE Z, et al., 2021). Entre todas as neoplasias pediátricas, cerca de 17 a 25\% ocorrem no SNC. Em países desenvolvidos, esses tumores são considerados os segundos mais frequentes, depois das leucemias. Já nos países em desenvolvimento, como o Brasil, os tumores do SNC correspondem à terceira neoplasia da infância (BENAGE SJ e PICKA MCM, 2016). Nos Estados Unidos da América (EUA), o câncer cerebral infantil já ultrapassou a leucemia e se tornou a causa mais comum de morte por câncer infantil. Além disso, os tumores cerebrais pediátricos de alto grau apresentam as maiores taxas de mortalidade por câncer em crianças (YE Z, et al., 2021).

As neoplasias cranianas pediátricas apresentam predominância no sexo masculino e nos indivíduos da raça branca, geralmente são graves e se destacam pela dificuldade de diagnóstico e tratamento precoce, mas, apesar de serem agressivas e invasivas, quando diagnosticadas precocemente possuem grande chance de eficácia em seu tratamento (BENAGE SJ e PICKA MCM, 2016). No entanto, esses tumores cerebrais resultam em desgaste psíquico, social e financeiro, gerando um profundo impacto, tanto nos pacientes como em suas famílias, sociedade e no sistema público de saúde, representando uma importante perda de potenciais anos de vida (CARVALHO WMO, et al., 2020).

Esse estudo teve por objetivo reunir informações recentes sobre tumores cerebrais na idade pediátrica, trazendo dados sobre as neoplasias mais comuns do sistema nervoso central nessa população, descobertas sobre seu desenvolvimento, consequências em qualidade de vida, tratamentos e diagnósticos.

\section{REVISÃO BIBLIOGRÁFICA}

O câncer pediátrico engloba um conjunto de doenças que possui em comum a proliferação descontrolada de células anormais, podendo ocorrer em qualquer topografia do organismo. Contudo, os tumores malignos pediátricos geralmente afetam as células do sistema hematopoiético e os tecidos de sustentação, o que se explica por serem predominantemente de natureza embrionária, oriundos de células indiferenciadas, diferentemente do que ocorre com o câncer em adultos, que costuma afetar as células do epitélio (CARVALHO WMO, et al., 2020).

Em crianças com câncer, características clínicas específicas como anomalias físicas, ocorrência de câncer em parentes jovens, histologias específicas de câncer e assinaturas únicas de mutação/metilação podem indicar a presença de uma síndrome de predisposição ao câncer subjacente. Porém, a proporção de crianças com um tipo de câncer que sugere uma predisposição entre todas as crianças com câncer ainda é desconhecida (NGUYEN TMK, et al., 2021).

Uma descoberta importante foi feita quando se identificou que a desregulação dos genes MYC, causando superexpressão de MYC ou estabilização de proteínas, é frequentemente encontrada em tumores cerebrais 
malignos, destacando seus papéis importantes como oncogenes. MYCN é um dos três membros da família MYC e é crucial para o desenvolvimento normal do cérebro. Está associado a um mau prognóstico em muitos tipos de tumores cerebrais pediátricos malignos. Ter como alvo terapias direcionadas ao MYCN provou ser um desafio devido à sua natureza intratável como um fator de transcrição e por sua importância na regulação de programas de desenvolvimento também em células saudáveis (BORGENVIK A, et al., 2021).

Meduloblastoma é considerado o tumor cerebral pediátrico maligno mais comum. Trata-se de um tumor embrionário que pode se diferenciar em células de linhagens neurais e é classificado como tumor grau IV pela Organização Mundial da Saúde (OMS) (ACHIHA T, et al., 2020). É altamente heterogêneo, podendo ser categorizado em quatro subgrupos moleculares principais: Hedgehog Sônico (SHH), Wingless (WNT), grupo 3 (G3) e grupo 4 (G4). Cada subgrupo manifesta características demográficas, patológicas e clínicas distintas, como idade, sexo, célula de origem, driver oncogênico, tendência de metástase, resposta à terapia convencional e prognóstico (WANG J, et al., 2020).

Entre os quatro subgrupos, o grupo 3 exibe o pior prognóstico, e possuem amplificação do MYC. Seu desfecho desanimador está associado ao alto índice de metástases e recorrência, diagnóstico tardio em estágio avançado da malignidade e regimes de quimioterapia e radiação se mostrarem menos eficazes. Portanto, precisa com urgência de uma nova terapia eficaz. Algumas estratégias terapêuticas epigenéticas direcionadas foram recentemente comprovadas para tratar efetivamente modelos pré-clínicos de G3, revelando uma direção promissora para investigação posterior (WANG J, et al., 2020).

O tratamento atual para meduloblastoma se resume à ressecção cirúrgica, radioterapia e quimioterapia. Os medicamentos direcionados têm mostrado privilégios únicos sobre as terapias citotóxicas tradicionais no equilíbrio entre eficácia e toxicidade, com muitos deles aprovados e amplamente usados clinicamente. Porém, apesar do progresso significativo existente em seu tratamento, uma parte das recidivas em crianças e a recorrência do tumor carregam um prognóstico ruim (WEN J e HADDEN MK, 2021).

Ainda sobre o meduloblastoma, os pesquisadores Achiha T, et al. (2020) explicam que a expressão e a função do Cluster de Diferenciação (CD) 166 ou molécula de adesão de células de leucócitos ativados (ALCAM), que é uma molécula transmembrana conhecida por ser um fator de adesão intercelular, nesse tipo de tumor ainda permanece obscura. Portanto, tiveram como objetivo esclarecer a importância e o papel funcional da expressão de ALCAM no meduloblastoma.

Para isso, Achiha T, et al. (2020) avaliaram a expressão de ALCAM em 45 pacientes com meduloblastoma e a relação entre sua expressão e o tipo patológico/subgrupo molecular. Oito ALCAM positivos (18\%), sete parcialmente positivos (16\%) e 30 casos negativos (67\%) foram detectados. Todos os sete casos do subgrupo molecular WNT foram ALCAM positivos e a expressão de ALCAM foi fortemente correlacionada com este subgrupo. Além disso, estudos funcionais usando linhas de células de meduloblastoma revelaram proliferação e migração afetadas pela expressão de ALCAM como um regulador positivo in vitro.

No entanto, o silenciamento de ALCAM não afetou a sobrevivência ou a formação de disseminação leptomeníngea em um modelo de camundongo ortotópico, mas induziu um fenótipo maligno com aumento da invasão de células tumorais nos locais de disseminação. Em conclusão, esses resultados revelaram que ALCAM exibiu expressão altamente específica no subgrupo WNT de meduloblastoma. Além disso, demonstraram que a cinética celular de linhagens de células de meduloblastoma pode ser alterada pela expressão de ALCAM (ACHIHA T, et al., 2020).

Outro tumor cerebral comum na população pediátrica é o glioma, que pode apresentar de grau I a IV, dependendo de sua malignidade. Os gliomas infantis incluem mais frequentemente os gliomas de baixo grau, sendo recomendado pela OMS distinguir entre gliomas difusos e tumores astrocíticos devido ao grupo substancialmente heterogêneo de gliomas de grau I - II. A classificação do glioma é relevante para a terapia, pois esta depende do conhecimento das variações moleculares subjacentes (JEAN-QUARTIER C, et al., 2021).

Cabe destacar que, apesar dos avanços médicos no diagnóstico e tratamento do câncer, o tratamento do glioma continua sendo basicamente a mesma abordagem, em todas as idades: cirurgia seguida de 
radioterapia e apenas ocasionalmente quimioterapia mais direcionada. Ainda assim, uma terapia bem tolerada por adultos pode não ser igualmente aplicável para um paciente pediátrico devido ao desenvolvimento contínuo do cérebro dessa população (JEAN-QUARTIER C, et al., 2021).

Segundo Fangusaro J, et al. (2021), os gliomas pediátricos de baixo grau são os tumores cerebrais mais comuns na infância. A sobrevida livre de progressão da doença é muito menor do que a sobrevida global, enfatizando a necessidade de tratamentos alternativos. A via óptica esporádica (sem neurofibromatose tipo 1) e o glioma hipotalâmico (OPHGs) são frequentemente recorrentes e causam déficits visuais significativos para seus portadores.

Em busca de tratamentos que priorizassem os resultados funcionais, Fangusaro J, et al. (2021) analisaram crianças com OPHGs recorrentes/progressivos tratadas em um estudo clínico de fase 2 avaliando a eficácia do medicamento selumetinibe, um inibidor MEK-1/2. Foram inscritos no estudo 25 pacientes, onde 6 (24\%) tiveram resposta parcial, $14(56 \%)$ tiveram doença estável e $5(20 \%)$ tiveram doença progressiva durante 0 tratamento. A sobrevida livre de progressão de dois anos foi de $78 \pm 8,5 \%$.

A acuidade visual melhorou em 4/19 pacientes (21\%), permaneceu estável em 13/19 (68\%) e piorou em apenas 2/19 (11\%). Cinco dos 19 pacientes (26\%) melhoraram os campos visuais e 14/19 (74\%) permaneceram estáveis. As toxicidades mais comuns foram elevação grau 1/2 da creatina fosfoquinase, anemia, diarreia, cefaleia, náusea/emese, fadiga, aumento de AST e ALT, hipoalbuminemia e erupção cutânea. Portanto, o selumetinibe foi tolerável e levou a respostas e estabilidade prolongada da doença em crianças com OPHGs recorrentes/progressivos (FANGUSARO J, et al., 2021).

Para aumentar a compreensão sobre informações genômicas dos gliomas de baixo grau, que são comuns na população infantil em geral e em indivíduos com a síndrome de predisposição ao câncer Neurofibromatose tipo 1 (NF1), Fisher MJ, et al. (2021) integraram dados clínicos, diagnósticos histológicos e análises genéticas/genômicas de vários níveis em 70 indivíduos de 25 centros oncológicos em todo o mundo. Seus resultados demonstraram que, enquanto a maioria dos tumores abrigava a inativação bi-alélica de NF1 como a única anormalidade genética, $11 \%$ apresentavam mutações adicionais.

Além disso, os tumores classificados como astrocitoma não pilocítico com base na análise de metilação do DNA eram significativamente mais propensos a abrigar essas mutações adicionais. A alteração secundária mais comum foi a mutação do gene FGFR1, que conferiu uma vantagem de crescimento adicional em vários modelos NF1 murinos experimentais complementares. Em conjunto, esta caracterização abrangente tem implicações importantes para o manejo de crianças com gliomas de baixo grau - NF1, distintas de suas contrapartes esporádicas (FISHER MJ, et al., 2021).

De acordo com van Schaik J, et al. (2021), sobreviventes de tumor cerebral na infância (STCI) correm o risco de desenvolver obesidade, o que influencia negativamente a saúde, especialmente a cardiometabólica. A prevalência de obesidade nessa população pode ter sido superestimada em estudos epidemiológicos anteriores devido à inclusão de crianças com craniofaringioma. E o grau de ganho de peso pode ter sido subestimado devido à exclusão de STCl que experimentaram ganho de peso, mas não estavam com sobrepeso nem eram obesos. É importante ressaltar que o ganho de peso pode ser um indicador de disfunção hipotálamo-hipofisária (HP) subjacente.

Diante dessas evidências, os pesquisadores objetivaram estudar a prevalência e os fatores de risco para ganho de peso significativo, sobrepeso ou obesidade e sua associação com disfunção de HP em uma amostra nacional de STCI não craniofaringioma e não hipofisária. A prevalência e os fatores de risco para ganho de peso significativo (variação do índice de massa corporal [IMC] $\geq+2,0$ pontuação de desvio padrão [SDS]), sobrepeso ou obesidade no acompanhamento e sua associação com disfunção de HP foram estudados (VAN SCHAIK J, et al., 2021).

De acordo com os resultados, de $661 \mathrm{STCl}$, com uma idade mediana de acompanhamento de 7,3 anos, $33,1 \%$ tiveram ganho de peso significativo, sobrepeso ou obesidade. Dos STCI entre 4 e 20 anos de idade, $28,7 \%$ estavam com sobrepeso ou obesos, em comparação com $13,2 \%$ da população geral entre 4 e 20 anos de idade. IMC SDS no diagnóstico, diagnóstico de glioma de baixo grau, diabetes insípido e puberdade 
precoce central foram associados com ganho de peso, sobrepeso ou obesidade. A prevalência de disfunção de HP foi maior em STCl com sobrepeso e obesidade em comparação com STCI de peso normal. Concluiuse que sobrepeso, obesidade e ganho de peso significativo são prevalentes na STCI. Um aumento no IMC durante o acompanhamento pode ser um reflexo da disfunção de HP, necessitando de vigilância endócrina mais intensa (VAN SCHAIK J, et al., 2021).

Recentemente, Tomomasa R, et al. (2021) relataram tumores de alto grau até então histopatologicamente inclassificáveis, sob o rótulo provisório de "tumores semelhantes a ependimoma com diferenciação mesenquimal (ELTMDs)", abrigando fusão C11orf95-NCOA1/2 ou -RELA. Genes de fusão recorrentes envolvendo C11orf95, C11 orf95-RELA, já foram identificados, porém apenas em ependimomas supratentorial entre tumores primários do SNC. Os pesquisadores examinaram as características clínico-patológicas e moleculares em cinco casos de ELTMDs. Com exceção de um caso de adulto (50 anos), todos os casos ocorreram em crianças de 1 a 2,5 anos. Todos os pacientes apresentaram uma lesão maciça no hemisfério cerebral.

De acordo com os dados trazidos por Tomomasa R, et al. (2021), embora os ELTMDs exibam associações morfológicas e genéticas com ependimoma supratentorial com C11orf95-RELA, eles não podem ser considerados como ependimoma. Sendo assim, mais análises de mais casos são necessárias para esclarecer suas diferenças e semelhanças.

Tanto a morbidade destas neoplasias como seu tratamento representam desafios para os pacientes, em decorrência dos déficits físicos, assim como as sequelas neuropsicológicas e neuroendócrinas que podem causar. Para reduzir as altas taxas de mortalidade dessas neoplasias pediátricas, se faz necessária a atuação de uma equipe especializada, multiprofissional, com oncologista, neurorradiologista, neurocirurgião, patologista e radioterapeuta. Além disso, através de um diagnóstico precoce é possível minimizar os índices de morbimortalidade que estão diretamente relacionados à doença (BENAGE SJ e PICKA MCM, 2016).

Fato é que avaliar tumores cerebrais pediátricos é frequentemente um desafio diagnóstico devido às suas diversas patologias tumorais, achados de imagem inespecíficos ou sobrepostos, artefatos de suscetibilidade de calcificação intratumoral ou hemorragia e artefatos de movimento em crianças. Nas últimas duas décadas, o advento das tecnologias de neuroimagem permitiu que os médicos detectassem a recorrência ou disseminação tumoral com maior certeza (YE Z, et al., 2021).

No entanto, essas descobertas foram desenvolvidas em grande parte para pacientes adultos com tumor cerebral, que são na maioria das vezes biologicamente distintos daqueles que ocorrem em crianças, que apresentam características genômicas e de imagem únicas. Os diagnósticos convencionais baseados em ressonância magnética também não oferecem informações adequadas sobre o tipo específico de tumor, grau do tumor, viabilidade do tumor e resposta ao tratamento das lesões (YE Z, et al., 2021).

Embora as técnicas avançadas de ressonância magnética (RM), como a imagem por tensor de difusão, a ressonância magnética de perfusão, a espectroscopia de RM e a imagem ponderada de suscetibilidade, sejam incorporadas aos protocolos clínicos de ressonância magnética, elas ainda são insuficientes. A abordagem de imagem de ressonância magnética multiparamétrica amplamente utilizada não reflete com precisão a histopatologia do tumor, como densidade celular tumoral, necrose, hemorragia ou bordas infiltrativas. Como estudos anteriores documentaram a heterogeneidade histológica e radiológica do tumor coexistindo em lesões tumorais de alto grau, é imperativo o desenvolvimento de uma técnica capaz de discernir a aparência variada dessas lesões de forma não invasiva (YE Z, et al., 2021).

Desde a última década até o momento, vários estudos relacionados à tomografia por emissão de pósitrons (PET) de O- (2- [18F] fluoroetil) -L-tirosina (18F-FET) em tumores cerebrais foram publicados na literatura. Assim, Muoio B, et al. (2018) fizeram uma análise de diversos estudos e afirmam que a 18F-FET PET pode ser útil no diagnóstico diferencial entre tumores cerebrais e lesões não neoplásicas e entre gliomas de baixo e alto grau. Também alegam que a integração do 18F-FET PET no planejamento cirúrgico permite um melhor delineamento da extensão da ressecção além das margens visíveis com a ressonância magnética padrão.

Para o planejamento da biópsia, a 18F-FET PET é particularmente útil na identificação de focos malignos em gliomas sem contraste. Também permite melhorar o planejamento da radioterapia em pacientes com 
gliomas. Este método pode ser útil para avaliar a resposta ao tratamento em pacientes com gliomas e melhorar o diagnóstico diferencial entre recorrência de tumores cerebrais e alterações pós-tratamento. A 18FFET PET pode fornecer informações prognósticas úteis em gliomas de alto grau. Em conclusão, a 18F-FET PET pode fornecer informações diagnósticas adicionais em comparação com a ressonância magnética padrão em neuro-oncologia (MUOIO B, et al., 2018).

Em outro estudo, Grosse F, et al. (2021) explicam que, como a ressonância magnética (RM) apresenta deficiências na diferenciação entre o tecido tumoral e as alterações pós-terapêuticas em pacientes com tumor cerebral pré-tratados, resolveram avaliar os benefícios da FET PET estática em pacientes com tumor cerebral pediátrico pré-tratados com resultados de RM convencionais equívocos. Para isso, avaliaram 22 FET-PET/TC estáticos de 17 pacientes pediátricos com tratamento multimodal.

Os resultados indicaram que a FET-PET/TC estática distinguiu de forma confiável entre tecido tumoral e alterações pós-terapêuticas em 16 de 17 pacientes. Identificou tecido tumoral vital corretamente em 13 pacientes e alterações pós-terapêuticas em três pacientes. Exceto no carcinoma do plexo coroide, todas as entidades tumorais mostraram aumento da captação de FET. Os autores concluiram que há um benefício claro de FET-PET/TC estática adicional quando a ressonância magnética convencional identificar lesões duvidosas em pacientes com tumor cerebral pediátrico pré-tratados. Esses resultados justificam estudos prospectivos que devem incluir varreduras dinâmicas (GROSSE F, et al., 2021).

\section{CONSIDERAÇÕES FINAIS}

Os tumores cerebrais malignos ocupam entre o primeiro e o terceiro lugar em incidência e mortalidade por câncer em crianças, dependendo do país, e são a principal causa de morte por câncer em crianças. No entanto, ainda pouco se sabe sobre a etiologia do tumor cerebral na infância. Entre os tumores cerebrais mais comuns na população pediátrica estão o meduloblastoma e o glioma. O uso de FET-PET parece ser mais preciso na diferenciação e avaliação de tumores cerebrais na idade pediátrica do que a ressonância magnética, devendo ser indicada quando houver dúvidas na identificação das lesões. Ainda se faz necessário um melhor direcionamento para os tratamentos desses tipos de câncer, o que vem sendo desenvolvido através de terapêuticas epigenéticas.

\section{REFERÊNCIAS}

1. ACHIHA T, et al. Activated leukocyte cell adhesion molecule expression correlates with the WNT subgroup in medulloblastoma and is involved in regulating tumor cell proliferation and invasion. PLoS One, 2020; 15(12): e0243272.

2. BENAGE SJ, PICKA MCM. A espectroscopia por ressonância magnética no diagnóstico de tumores encefálicos pediátricos. Tekhne e Logos, 2016; 7(3): 100-113.

3. BORGENVIK A, et al. Targeting MYCN in Molecularly Defined Malignant Brain Tumors. Frontiers in Oncology, 2021; 28; 10:626751.

4. CARVALHO WMO, et al. Aspectos epidemiológicos do câncer infantojuvenil em uma capital do nordeste brasileiro. Revista Eletrônica Acervo Saúde (REAS), 2020; 2(11): e4045.

5. FANGUSARO J, et al. A Phase 2 trial of selumetinib in children with recurrent optic pathway and hypothalamic lowgrade glioma without NF1: A Pediatric Brain Tumor Consortium Study. Neuro Oncology, 2021; 25: noab047.

6. FISHER MJ, et al. Integrated molecular and clinical analysis of low-grade gliomas in children with neurofibromatosis type 1 (NF1). Acta Neuropathologica, 2021; 14.

7. GROSSE F, et al. Benefit of static FET PET in pretreated pediatric brain tumor patients with equivocal conventional MRI results. Klinische Padiatrie, 2021; 17.

8. JEAN-QUARTIER C, et al. Mutation-based clustering and classification analysis reveals distinctive age groups and age-related biomarkers for glioma. BMC Medical Informatics and Decision Making, 2021; 21(1): 77.

9. MUOIO B, et al. Recent developments of 18F-FET PET in neuro-oncology. Current Medicinal Chemistry, 2018; 25(26): 3061-3073.

10. NGUYEN TMK, et al. Proportion of children with cancer that have an indication for genetic counseling and testing based on the cancer type irrespective of other features. Familial Cancer, 2021; 26.

11. SHAHAB S, FANGUSARO J. Neonatal central nervous system tumors. Clinics in Perinatology, 2021; 48(1): 35-51.

12. TOMOMASA R, et al. Ependymoma-like tumor with mesenchymal differentiation harboring C11orf95-NCOA1/2 or RELA fusion: A hitherto unclassified tumor related to ependymoma. Brain Pathology, 2021; 12: e12943. 
13. VAN SCHAIK J, et al. High Prevalence of Weight Gain in Childhood Brain Tumor Survivors and Its Association With Hypothalamic-Pituitary Dysfunction. Journal of Clinical Oncology, 2021; 23: JCO2001765.

14. WANG J, et al. Effective inhibition of MYC-amplified group 3 medulloblastoma by FACT-targeted curaxin drug CBL0137. Cell Death \& Disease, 2020; 11(12): 1029.

15. WEN J, HADDEN MK. Medulloblastoma drugs in development: Current leads, trials and drawbacks. European Journal of Medicinal Chemistry, 2021; 215: 113268.

16. YE Z, et al. Diffusion histology imaging differentiates distinct pediatric brain tumor histology. Scientific Reports, 2021; 11(1): 4749. 